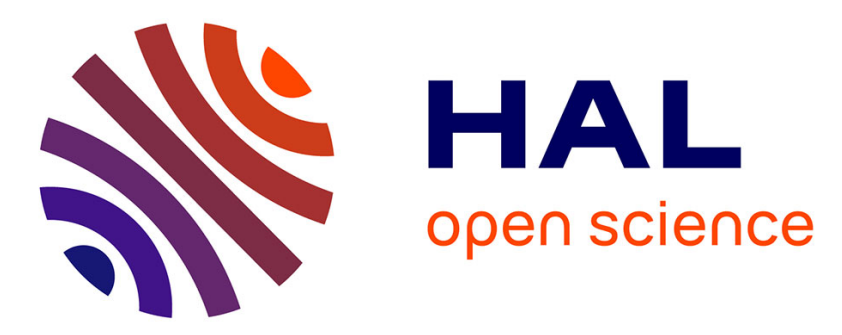

\title{
Games that Make Curious: An Exploratory Survey into Digital Games that Invoke Curiosity
}

\author{
Marcello A. Gómez Maureira, Isabelle Kniestedt
}

\section{To cite this version:}

Marcello A. Gómez Maureira, Isabelle Kniestedt. Games that Make Curious: An Exploratory Survey into Digital Games that Invoke Curiosity. 17th International Conference on Entertainment Computing (ICEC), Sep 2018, Poznan, Poland. pp.76-89, 10.1007/978-3-319-99426-0_7 . hal-02128582

\section{HAL Id: hal-02128582 \\ https://hal.inria.fr/hal-02128582}

Submitted on 14 May 2019

HAL is a multi-disciplinary open access archive for the deposit and dissemination of scientific research documents, whether they are published or not. The documents may come from teaching and research institutions in France or abroad, or from public or private research centers.
L'archive ouverte pluridisciplinaire HAL, est destinée au dépôt et à la diffusion de documents scientifiques de niveau recherche, publiés ou non, émanant des établissements d'enseignement et de recherche français ou étrangers, des laboratoires publics ou privés. 


\title{
Games That Make Curious: An Exploratory Survey into Digital Games That Invoke Curiosity
}

\author{
Marcello A. Gómez Maureira ${ }^{1}$ and Isabelle Kniestedt ${ }^{2}$ \\ 1 Leiden Institute of Advanced Computer Science, Niels Bohrweg 1, Leiden, The \\ Netherlands m.a.gomez.maureira@liacs.leidenuniv.nl \\ 2 Delft University of Technology, Jaffalaan 5, Delft, The Netherlands \\ i.kniestedt@tudelft.nl
}

\begin{abstract}
Curiosity is an important aspect of life, but studying it is challenging without reliable stimuli. Digital games provide an ideal stimulus to investigate the circumstances that trigger curiosity and how it is expressed. A survey was conducted with the goal of assessing what game titles and game genres should be analysed to further the study of curiosity. To consider different types of curiosity, we included the Five-Dimensional Curiosity Scale (5DC) questionnaire. The survey was completed by 113 participants, and resulted in 301 game suggestions that warrant further analysis. Exploration, social simulation, and collecting tasks within games were found to rank high in triggering curiosity. We further found that social curiosity in individuals correlates with having curiosity triggered by social simulations.
\end{abstract}

Keywords: Game user research · Curiosity research · Game analysis.

\section{Introduction}

Curiosity plays a crucial role in many aspects of human life. It is a sign for intrinsic motivation to learn and explore. In education and research, curiosity is frequently credited as one of the most important factors for progress. It is equally high regarded as motivator for creative endeavours, ingredient for stimulating communication, and sign for personal well-being. As a concept that involves both behavioural and emotional components, studying curiosity is challenging. However, recent work has made progress in establishing definitions of curiosity and psychometric instruments to measure it. As a result, there is growing interest in the applied use of what has been learned about curiosity, such as to improve teaching methods $[24,11]$ and the design of video games [31,26]. Games, here understood as systems for structured play, provide multi-faceted environments for stimulating curious behaviour. At the same time, there is a lack of specific knowledge on which games or game genres stand out in their ability to invoke curiosity. Knowing this would allow for more in-depth analysis of the methods that existing games use to make players curious.

In this study we thus aim to lay the groundwork for filling this gap with an exploratory survey involving 113 participants. In the survey, we asked players to 
rank well-known games according to how curious they felt while playing them. We further inquired which game titles made them curious in the past, using established dimensions of curiosity as prompts (see section 3.3). The individual dimensions, described in detail in section 3.1, give a more nuanced account of an individual's curiosity than assessing it on a single scale. A total of 301 games were mentioned by participants and were then categorized according to a list of pre-defined game genres. This categorization allowed us to analyse patterns within the varied selection. With this, we examine what games and game genres are successful in invoking curiosity. As final part of the survey, participants filled in the 5DC questionnaire [13]. This empirically validated questionnaire establishes a score for each participant according to five dimensions of curiosity (see section 3.1). With this data, we examined whether there is a connection between an individual's tendency to become curious and the game genres that invoke curiosity.

The primary contribution of this study takes the form of an informed selection of games and game genres that warrant closer analysis in regards to how the elicitation of curiosity may be designed for within a game. We also looked for results that would help inform the starting point of this analysis and impacts of personality dimensions on the games that invoke an individual's curiosity. Due to its exploratory nature, this work does not provide sufficient data to formulate a generalizable theory. Nonetheless, we believe that it is an important step in exploring curiosity within different games and a basis for further work in this direction.

\section{Related Work}

Most research efforts regarding curiosity have taken place in the fields of philosophy $[9,27]$ and psychology $[6,1]$. Inherent in this past is the fact that definitions of curiosity vary, ranging from accounts of human aspirations to describing it as instigating stimulant for interaction with the environment.

In this study we understand curiosity as an intrinsic motivation for pursuing new knowledge and experiences that is accompanied by pleasure and excitement. This understanding of curiosity is based on a meta review of academic articles which aimed to find commonalities in prior research [7]. In the review, the author discusses different research lenses through which curiosity has been studied. These lenses do not necessarily contradict each other, but focus on different aspects of curiosity. One view of curiosity, for example, is to consider it a primal drive that requires satisfaction [1,2], not unlike satisfying hunger [27]. Another view is to see curiosity as a need to fill gaps in knowledge [21], requiring both existing knowledge to be aware of such a gap, as well as the evaluation that the gap is neither too large nor too insignificant to be filled [30]. Important for our study is the differentiation between curiosity as a state and curiosity as a trait. The former is the 'in-the-moment' drive for exploratory behaviour and its emotional impact [21]. Trait curiosity, on the other hand, is an individual's tendency or disposition to become curious and is considered a relatively stable personality 
trait [20]. It should be noted that studies have shown an influential relationship between trait and state curiosity $[18,12,25]$.

Most of the existing work in quantifying curiosity is concerned with measuring trait curiosity $[19,17]$ or related personality traits, such as intrinsic motivation $[5$, 22] or sensation seeking [33]. To quantify curiosity in our study, we follow the curiosity model proposed by Kashdan et al. which suggests the involvement of five dimensions to describe an individual's disposition to become curious [13]. The individual dimensions were selected based on preceding work and validated through three surveys. The result of their study is the 'Five-Dimensional Curiosity Scale' (5DC) which quantifies trait curiosity through a validated questionnaire. We describe the individual dimensions of the 5DC in section 3.1.

Games are an interesting area for researching curiosity, as they introduce further related concepts that can help to gain a better understanding. Costikyan's work regarding the role of uncertainty in games, for example, involves curiosity and describes it as an important motivator to engage in gameplay [4]. For Klimmt [14], curiosity is part of a conceptual model for player engagement, i.e. the reason for why people choose to play games. Studies into player profiling seek to establish player archetypes that involve personality traits and motivations, including curiosity [26]. In these cases, curiosity is not studied on its own but mentioned as a contributing factor. Games have also been proposed as instruments for measuring curiosity, as was done in a study from 2012 to measure scientific curiosity in children [10]. In this experiment, the performance of players within an exploration game was used as a behavioural measure instead of relying on self-report through a questionnaire.

An improved understanding of curiosity also benefits efforts in understanding player experience and can inform game development. Research by To et al. [31] investigated how game designers can elicit the curiosity of players. In their study, they follow a model of curiosity [15] that distinguishes between different triggers of curiosity. This approach is particularly useful for creating generalizable design guidelines, as it gives game designers a range of possible design interventions for invoking curiosity. Overall, existing research shows that games are able to elicit curiosity, and that this ability is useful for both research and development. We are not aware of work that investigates which games stand out as being particularly capable of invoking curiosity, and thus aim to provide such insights with this study.

\section{Research Design}

To gather data from a large number of participants we used an online survey aimed at people playing video games. The survey link was distributed on Facebook groups connected to gaming and game research, and included the following modules: Demographics, shared selection of games, suggestions by curiosity dimensions, and the 5DC questionnaire. We describe each of the modules below, as well as the formulation of genres to categorize games suggested by participants. 


\subsection{DC Questionnaire}

We used the 5DC questionnaire [13] to explore if the individual dimensions of trait curiosity predict the game genres that invoke curiosity in a player. It involves 25 questions, rated on a 5-point Likert scale, and results in scores on five dimensions: Joyous Exploration (JE) - being motivated by novelty, Deprivation Sensitivity (DS) - need of resolving, Stress Tolerance (ST) - ability to cope with uncertainty, Social Curiosity (SC) - wanting to know about others, and Thrill Seeking (TS) enjoyment of anxiety.

The questionnaire has been developed by selecting items of existing measures that evaluate interest and curiosity, openness to experience, need for cognition, boredom proneness, and sensation seeking. The individual items were evaluated through three studies with a combined sample size of 3911 participants. The resulting questionnaire is not limited to a specific demographic, but has been evaluated with a representative sample of the U.S. population. Finally, the questionnaire was examined in regards to test-retest reliability through a 4month follow-up, with results being within the range of stable personality traits.

\subsection{Shared Selection of Games}

Players were asked to rank games they had played out of a selection of 15 acclaimed game titles, in order to explore which of the games invoked curiosity while playing (see Figure 1). It should be noted that this study is not about how curious people are to play a specific game (e.g. not played yet, but curious to do so), but how curious they felt as part of the gameplay. Any question regarding curiosity was phrased to reflect this focus. By presenting a predefined list we could collect data on specific game titles that can be considered of solid quality in terms of design. The measure for quality is provided by a game's Metacritic score [23], which itself is comprised of the evaluation of several game critics. While this measure is based on subjective evaluations, it is a productive approximation for choosing games of comparable quality. We took the top 15 games listed on Metacritic, after restricting our selection to games that were released in the last 10 years and combining games of the same series that met that criteria. The resulting selection involved games with a Metacritic score of 94 or higher (out of 100).

\subsection{Suggestions by Curiosity Dimensions}

Given that we are interested in exploring what game titles should be analysed in regards to their ability to invoke curiosity, we asked participants to suggest game titles. In order to consider different dimensions of curiosity, we used five categories for which suggestions could be added (up to two games per category). These were described as "Games that ...": "let me explore or find out new things" (GEXP), "let me solve something" (GSOL), "let me feel safe and stress-free" (GSAF), "let me understand people or let me connect to people" (GCON), and "make me feel excited and alive" (GALI). 
We based the category descriptions on the questions that make up the dimensions of the 5DC questionnaire, rather than the descriptions of the categories themselves as defined by the researchers. As a result, the categories should match the five curiosity dimensions: GEXP matching JE, GSOL $=\mathrm{DS}, \mathrm{GSAF}=\mathrm{ST}$, GCON $=\mathrm{SC}$, and GALI $=$ TS. In addition to suggesting game titles, participants ranked the games they provided in order of how curious they felt while playing them.

\subsection{Ranking}

At this point we should note how the ranking of game titles is evaluated, and why we chose to let participants rank rather than rate them on a scale. Reporting about affective constructs is challenging, especially if it has to be mapped to a numerical expression. Likert scales that measure such constructs often use short phrases against which a scale number can be compared. It can, however, be difficult to consistently apply such a mapping [32]. Ranking alleviates this problem as it allows participants to use the individual items as points of reference. The challenge is then how to evaluate such rankings across participants. While some participants play the same game titles, a lot more do not. Likewise, while some rank a large number of items, others only rank a few, either because they have not played as many games or do not consider them as invoking curiosity. To address this challenge, we implemented the TrueSkill rating system, developed by Microsoft for ranking and match-making on their Xbox LIVE online platform [8]. Available as a Python package [16], TrueSkill uses a Bayesian inference algorithm that updates the score of individual match items (usually representing the skill of players) every time a match is played. Since score-points can be lost, participating in a high number of matches (i.e. having been played by many participants) does not necessarily result in a higher ranking. As such, we can use this algorithm to compare items (the individual games) that vary in regards to how often they were mentioned. To use TrueSkill, we paired up all combinations within a ranking to create 'match-ups', taking the rank as deciding factor on which item 'wins' the match. After matching up all possible combinations, we used the resulting score as a measure for both the rank of an item, as well as the relative distance to other items. While the score is an arbitrary number, it can be used in relation to other scores. Items that have relatively similar scores can then be considered closer to equal, while those that differ by wide margins are likely to have 'won' a large number of comparisons. While using the TrueSkill algorithm provides a useful model for ranking items, we cannot evaluate how significant the resulting rank is. To our knowledge there is no statistical test that could be consulted to estimate how representative the overall rankings are for a larger population. While a different survey design would have remedied this, it would mean to either only include participants that have played the same games, or resort to rating games on a scale. 


\subsection{Formulation of Game Genres}

When asking participants to suggest game titles, we can expect a wide range of games. This makes it difficult to explore general patterns, as the number of participants that will have played the same games will be limited. In order to capture the most defining aspects of a game instead, we assigned two game genres to each of the suggested games. The challenge of involving genres is the lack of a shared definition. Genre classifications can originate from multiple motivations, such as easing retrieval of titles, academic efforts of building a taxonomy, or marketing considerations [3]. For this reason we devised a list of 11 game genres based on commercially used genres, but qualified by a statement that defines the genre in our study. Some of the more commonly used genres have been omitted or modified to suit the goals of this study. As an example, "Action" can be a problematic genre, as a large number of games involve fast-paced sequences but may be based on vastly different game mechanics. An additional challenge is that games frequently involve a wide range of game genres. Grand Theft Auto $V$ lets players shoot virtual characters, race cars, but also allows them to ride a roller-coaster, perform yoga, and solve a murder mystery. By attributing the genres Reflex and Exploration, some nuance is undoubtedly lost. While imperfect, this approach still provides a tentative measure for evaluating which actions performed in a game can be conductive to invoking curiosity. We assigned the following genres:

- Reflex - requires fast reflexes to perform well.

- Exploration - provides spatial or conceptual discovery that is not automatically brought to the attention of the player.

- Puzzle - presents tasks that must be solved through predefined processes.

- Strategy - requires players to plan their actions in advance, taking into consideration available resources.

- $R P G$ - defined by assuming the role of one or more characters and making choices that impact game progression.

- Story - progresses as part of a structured narrative.

- Task Sim - asks players to perform tasks that are associated with professions, emphasizing the nature of the task.

- Social Sim - asks players to perform tasks associated with social interactions and everyday tasks.

- Collecting - is structured around gathering items for the purpose of having gathered all or as many items as possible.

- Frantic - uses aesthetic elements and/or concurrent game mechanics to saturate the cognitive capabilities of players.

- Chance - progress in the game is largely independent from the actions taken by the player, but differs between game sessions.

\section{Procedure}

The survey was conducted over a period of one month during which 117 participants completed the survey. Participants were recruited through convenience 


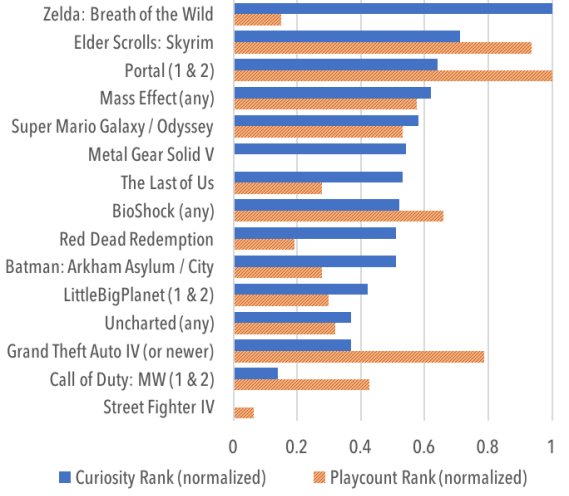

Fig. 1. Shared selection of games ranked by how curious participants felt and how many participants had played them. Values are normalized to $0-1$ for comparison ( $0=$ lowest rank, $1=$ highest rank).

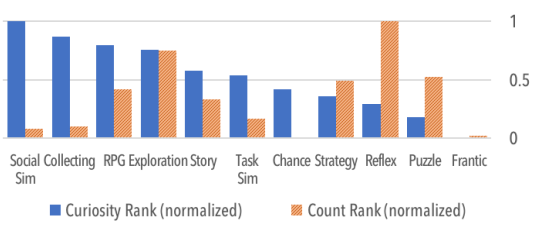

Fig. 2. Game genres ranked by participants' curiosity and how often it was mentioned.

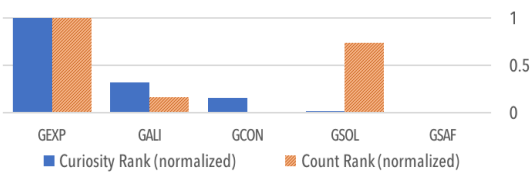

Fig. 3. Curiosity categories participants' curiosity and how many games were mentioned.

sampling and referral sampling. The target demographic included everyone who does or has played video games at some point in their lives. While we did not track the nationality of participants, the most likely audience was English-speaking people in Western Europe and the U.S. The first part of the survey asked questions about demographics. Items for playing frequency were: Every day, Every week, Occasionally, Rarely, and Never - the survey ended for those who chose 'Never'. The second part asked which of 15 games they had played. If two or more games were selected, the next page asked participants to rank the games they had played in terms of how much they had triggered their curiosity while playing. The third part asked participants to provide up to two games (entered as free text) for each of the five curiosity dimensions (see section 3.3). If participants provided two or more items overall, they were asked to rank those games. Participants were free to rank any number of items in both rankings, including none. The final part of the survey included the 25 questions of the 5DC questionnaire. Each game provided by participants was assigned two game genres in order of importance. To determine which game genres should be assigned, the authors individually assigned game genres in accordance to the definitions (see section 3.5). Assigned game genres were then compared for each game, and disagreements were resolved through discussion.

\subsection{Data Processing}

A text matching algorithm [28] was used to sort game titles that were entered as free text. The resulting list was checked for misspellings and corrected if necessary. Abbreviations were adjusted manually, and entries that could not be resolved were removed from the dataset. Entries belonging to the same game 
series, or referring to the same game by another name, were combined into a single entry (e.g. Oblivion becomes Elder Scrolls), with the exception of Zelda: Breath of the Wild (separate from Zelda) and World of Warcraft (separate from Warcraft). While this makes it impossible to consider elements of the individual games, games in a series tend to share many of the same general mechanics. This decision allowed us to examine the games over a larger sample size, in service of our exploration of general patterns in game design. Since Zelda: BotW and WoW show significant departures from their predecessors, these titles were retained. To identify correlations between the rankings and dimensions on the 5 DC questionnaire, three ranks were created for each participant: Shared selection ranking, game genre ranking, and curiosity category ranking. Shared selection ranking ranged from 1 to 15 , as a maximum of 15 games could be ranked. A game that was ranked as most curiosity invoking was given rank 1, followed by 2 , continuing to rank 15. Games that a participant had not played, or had not ranked, were given the lowest possible rank of 15 .

To create ranks by genre, every ranked game title was assigned two genre labels. With 11 genres, the ranking for each ranged from 1 to 11. Since game titles at different ranks could involve the same genres, a TrueSkill rating was calculated for every genre that was part of a participant's ranking. The rating was calculated by comparing all possible genre combinations within a participant's ranking, using both the rank and whether the genre was the primary or secondary label. The genre with the highest rating was ranked 1, followed by lower rated genres. Genres that were not used received the lowest possible rank of 11. In addition to creating this ranking for each participant, an overall ranking across all participants was created as well.

Curiosity category ranking closely followed the procedure for game genre ranking, with the difference that each game title represented a single category: the category under which the game title was entered. Possible ranks ranged from 1 to 5 , reflecting the number of curiosity categories used to represent the five curiosity dimensions of the 5DC questionnaire. Again, an overall ranking was created in addition to per-participant rankings. Finally, 5DC questionnaire scores were created for each participant by calculating the mean of Likert scale ratings of questions contributing to one of the five dimensions. Likert scale ratings were reverse scored for the Stress Tolerance (ST) dimension, as required by the questionnaire.

\section{$5 \quad$ Results}

Out of 117 participants, 113 played video games at least 'Rarely', and thus completed the survey ( $38.9 \%$ female, mean age $\mathrm{M}=27.64, \mathrm{SD}=5.8)$. The mean playing frequency was $\mathrm{M}=2.0(\mathrm{SD}=0.94)$, which corresponds to 'Every week'.

The overall ranking of the shared game selection is shown in Figure 1, with TrueSkill ratings normalized to a $0-1$ range. The count ranged from 19 for Metal Gear Solid V (normalized to 0) to 66 for Portal (normalized to 1). To evaluate the quality of the ranking, all 105 possible combinations of pair-wise rank comparisons 


\begin{tabular}{|c|c|}
\hline Game Category & Game Titles (Number of Mentions) \\
\hline $\begin{array}{l}\text { GEXP } \\
\text { (Explore, find out) } \\
92 \text { unique titles }\end{array}$ & $\begin{array}{l}\text { Elder Scrolls (17), Fallout (14), Minecraft (11), } \\
\text { Zelda: BotW (9), Dark Souls (8), Horizon: Zero } \\
\text { Dawn (8), The Witcher (8), Subnautica (7), } \\
\text { World of Warcraft (7), Final Fantasy (5), } \\
\text { Assassin's Creed (5), Zelda (5) }\end{array}$ \\
\hline $\begin{array}{l}\text { GSOL (Solve) } \\
113 \text { unique titles }\end{array}$ & $\begin{array}{l}\text { Portal (29), The Witness (8), Elder Scrolls (7), } \\
\text { Myst (5), The Talos Principle (5) }\end{array}$ \\
\hline $\begin{array}{l}\text { GSAF (Safe, stress-free) } \\
100 \text { unique titles }\end{array}$ & $\begin{array}{l}\text { Sims (8), Stardew Valley (7), Elder Scrolls (6), } \\
\text { Cities: Skylines (5) }\end{array}$ \\
\hline $\begin{array}{l}\text { GCON (Connect to people) } \\
84 \text { unique titles }\end{array}$ & $\begin{array}{l}\text { World of Warcraft (13), Final Fantasy (7), } \\
\text { Journey (5), Sims (5) }\end{array}$ \\
\hline $\begin{array}{l}\text { GALI } \text { (Excited, } \\
\text { feeling alive) } \\
108 \text { unique titles }\end{array}$ & $\begin{array}{l}\text { GTA (7), PlayerUnknown's Battlegrounds ( } 6 \text { ), } \\
\text { World of Warcraft (5), Horizon: Zero Dawn ( } 5 \\
\text { Elder Scrolls }(5)\end{array}$ \\
\hline
\end{tabular}

Table 1. Game titles mentioned for each of the five curiosity categories (showing titled with at least 5 mentions). Titles in bold appear in multiple categories.

\begin{tabular}{lllrrr}
\hline 5DC Dimension & - & Measure & rho & p & VS-MPR \\
\hline JE & - & RPG & 0.2 & 0.037 & 3.019 \\
Joyous Exploration & - & GCON & 0.234 & 0.016 & 5.617 \\
\hline DS - Deprivation Sensitivity & - & Collecting & -0.193 & 0.045 & 2.655 \\
\hline & - & GTA (IV+) & 0.252 & 0.007 & 10.607 \\
& - & Zelda: BotW & -0.239 & 0.011 & 7.499 \\
ST & - & Call of Duty & 0.214 & 0.023 & 4.295 \\
Stress Tolerance & - & RPG & 0.293 & 0.002 & 29.545 \\
& - & Puzzle & -0.226 & 0.018 & 5.037 \\
\hline SC & - & GSOL & -0.222 & 0.022 & 4.310 \\
Social Curiosity & - & Social Sim & 0.220 & 0.022 & 4.414 \\
\hline TS & - & Frantic & -0.212 & 0.03 & 3.535 \\
Thrill Seeking & - & GTA (IV+) & 0.279 & 0.003 & 22.456 \\
\hline
\end{tabular}

Table 2. Two-tailed Spearman's rank correlations between 5DC dimensions and other measures. VS-MPR shows maximum possible odds in favour of $\mathrm{H}_{1}$ [29].

(involving only participants who had played both games) were checked. Out of these, 7 combinations (6.7\%) did not confirm the ranking, while the rest did. For the game suggestions per category module, a total of 301 unique game titles were mentioned. Table 1 shows which game titles were mentioned most frequently for each of the five game categories. Figure 3 shows the TrueSkill ranking of the five categories with measures normalized to a $0-1$ range. In terms of counts, GSAF had the fewest game suggestions (103, normalized to 0), and GEXP had the most (180, normalized to 1$)$. The overall TrueSkill ranking of game genres associated with games provided by participants is shown in Figure 2. The frequency of game genres used ranged from 10 for Chance (normalized to 0), to 318 for Reflex (normalized to 1).

The aggregated results of the $5 \mathrm{DC}$ questionnaire were: $\mathrm{JE}(\mathrm{M}=5.38, \mathrm{SD}=0.86)$, $\mathrm{DS}(\mathrm{M}=4.98, \mathrm{SD}=1.15), \mathrm{ST}(\mathrm{M}=4.36, \mathrm{SD}=1.42), \mathrm{SC}(\mathrm{M}=5.11, \mathrm{SD}=1.14)$, TS $(\mathrm{M}=4.20, \mathrm{SD}=1.34)$ - each based on Likert scale ratings from 1 to 7 . We note that we use a significance level of 0.05 for all statistical tests in this study. Significant correlations between 5DC dimensions and rankings are shown in Table 2. For the purpose of clarity, rho was inverted to match the meaning of an increase in score in the individual 5DC dimensions (that is, a rating of 1 in a ranking is 'higher' than a 2 , but 1 is lower than 2 in the $5 \mathrm{DC}$ questionnaire).

In terms of demographics, playing frequency differed between genders (MannWhitney $\mathrm{U}=1987, \mathrm{p}=0.004$, two-tailed), with male participants playing more frequently. Further differences were found in the ranking of the game genres Strategy (U=1911, $\mathrm{p}=0.002$, two-tailed, lower ranking in females) and Task Sim ( $\mathrm{U}=1714, \mathrm{p}=0.036$, two-tailed, lower ranking in females). Looking at differences in scores of curiosity dimensions, ST was significantly higher in males ( $U=978$, $\mathrm{p}=0.001$, two-tailed), while SC was significantly higher in females $(\mathrm{U}=1988$, $\mathrm{p}=0.006$, two-tailed). Participants' age was found to be correlated with a lower score of the 5DC dimension Social Curiosity (rho $=-0.297, \mathrm{p}=0.001$ ), a lower ranking of the game category GSAF ( $\mathrm{rho}=-0.231, \mathrm{p}=0.018)$, and with a higher ranking of the Puzzle genre $(\mathrm{rho}=0.226, \mathrm{p}=0.019)$. 


\section{Discussion}

In the following section we discuss the implications of our results. First, we identify the game titles and genres that warrant further attention. We then look at correlations between the games, game genres, and the curiosity dimensions. Finally, we examine the connection between the curiosity-based categories of suggested games and the 5DC curiosity dimensions.

\subsection{Games and Genres for Analysis}

Participants offered a wide range of games when asked to suggest titles for each of the five curiosity categories. As shown in Table 1, several titles were mentioned by more than one person. Most of these games gravitated towards one of the five categories (e.g. Minecraft in GEXP). For these games, further analysis towards curiosity invoking design should focus on the theme of the category. Some games (shown in bold) span multiple categories and, for this reason, should be examined in regards to how multiple kinds of curiosity can be motivated in harmony. From the shared selection of games, we decided to specifically focus on those that ranked 0.6 or higher: Zelda: Breath of the Wild (Z:BotW), The Elder Scrolls: Skyrim, Portal 1 \& 2 2, and the Mass Effect series. Of these games, the defining game genres are Exploration, Puzzle, RPG, and Reflex. Another interesting pattern to consider is games that have not been played by many participants, but ended up high in the ranking. This includes Z:BotW, Metal Gear Solid V, The Last of Us, Red Dead Redemption, and Batman: Arkham Asylum/City. The game genres of these games consist of Exploration, Reflex, Strategy, Puzzle, and Story. In both patterns we can see that Exploration and Reflex seem to be involved in games that rank high in curiosity elicitation. For Exploration this is also reflected in the ranking of game categories derived from the 5DC dimensions (see Figure 3). When asking participants to rank the games they provided, the GEXP category ranked far above other categories, suggesting that exploration and "finding out new things" are considered dominant aspects of what elicits curiosity in a game. Next, we examined potential correlations between the participants' scores on trait curiosity and the shared selection of games. GTA was ranked higher by participants with increased Stress Tolerance and Thrill Seeking, while Call of Duty $(\mathrm{CoD})$ was ranked higher with increased Stress Tolerance. Given that both $G T A$ and $C o D$ were ranked low overall, this could mean that players do not consider these dimensions as defining of what elicits their curiosity. $Z$ :Bot $W$ was ranked higher with decreasing ST. Here as well, given the high rating of $Z: B o t W$, stress tolerance does not seem to be a predictor of overall curiosity. We speculate that, despite having combat and potentially stressful elements, $Z$ :Bot $W$ allows players that are easily stressed to still express their curiosity. On the other hand, to express curiosity in GTA or CoD, players need a higher stress tolerance.

Turning to the game labels, we note several interesting results. Social Sim and Collecting stand out as genres that were part of only a few games, but ended up at the top of the ranking. These genres, and the suggested games that had this genre assigned to them, should be analysed more closely to see how games 
can benefit from such elements in terms of increasing their potential to invoke curiosity. Reflex and Puzzle are the opposite of these categories, as they ranked low in curiosity despite being present often. This is interesting as Deprivation Sensitivity specifically deals with puzzle-like stimuli. Games suggested under the corresponding category (GSOL) are mentioned frequently, but ranked low in curiosity (see Figure 3). It could be that this dimension of curiosity does not strike players as an important component of curiosity. Interestingly, both $Z: B o t W$ and Portal rank high in the shared game list, despite carrying the Puzzle genre. For these games, it may not be the fact that they include puzzles that invokes curiosity in players. Instead, we hypothesise that exploration is a more defining component in $Z: B o t W$, whereas Portal stands out through an unusual base mechanic and surprising narrative components. Overall, we speculate that game genres that strike a balance between uncertainty and structure tend to rank high, while genres that are highly deterministic (requiring cognitive or physical aptitude) or highly random tend to rank lower in curiosity.

Looking at correlations between game labels and trait curiosity dimensions, $R P G$ stands out as the only genre that correlates positively with several dimensions: Joyous Exploration, Stress Tolerance, and Thrill Seeking. It should be noted that these dimensions have been found to correlate with each other. However, given that other game genres correlate with only one of these dimensions, it is likely that $R P G$ invokes curiosity across several dimensions through its gameplay. As a genre, $R P G$ is less defined by a specific sort of gameplay and rather by how game actions are carried out and contextualized by the player. It can be argued that $R P G$ is a game genre that reflects a sense of going on an adventure, encapsulating both elements of discovery and physical excitement.

Another interesting finding is Social Curiosity, which correlates with high ranking of Social Sim, as well as low ranking of Frantic games. The associated game category (GCON) was not ranked high, and did not correlate with Social Curiosity. While we expected to find a positive correlation, the actual finding does makes sense. The phrasing of the category ("Games that let me understand people or let me connect to people") can be interpreted as a physical affordance, instead of a more conceptual social connection to, for instance, in-game characters. Given that Social Sim ranked highly among game genres, social simulation games and socially oriented curiosity should be analysed closely for their ability to make players curious. This is especially important given that Social Curiosity was the only dimension not correlated to another dimension, which suggests a certain uniqueness.

\subsection{Impact of Curiosity Dimensions}

Few correlations were found between the created game genres and the corresponding dimensions of the 5DC. A possible explanation for this could be that participants have strong associations with the terms that were used (e.g. "explore or find out") that do not match the definitions of the curiosity dimensions. 'Exploration', for example, is often understood within games to be spatial in nature, i.e. exploring an environment. The 5DC questions on the other hand are more 
focused on the exploration of knowledge and (challenging) stimuli. Similarly, the GCON category asked participants to list games related to connectedness and understanding people. Being connected in games, however, might not necessitate social curiosity. At present, the only curiosity dimension that corresponded with a directly similar game genre was Social Curiosity (with Social Sim). Besides this, while we did not find the direct correlations between game categories and trait dimensions that we would expect to see, we do see plausible connections between dimensions, game genres and individual games. It could therefore be that connections exist, but were not detected due to decisions in our research design. Despite our efforts in basing the game categories on the 5DC questions, a rephrasing might elicit game suggestions more in line with the curiosity dimensions. Another possibility is that trait curiosity is not (fully) predictive of what makes a person curious within a game.

\section{Conclusion}

The aim of this study was to provide a starting point for the consideration of what game titles and genres should be analysed in regards to their potential to invoke curiosity. Through the suggestions of survey participants, we have gathered such a collection and established a ranking of critically acclaimed games for further study. We further investigated what game genres were likely to be ranked high in triggering curiosity, and have looked at how they correspond to individual dimensions of trait curiosity. We found that games involving exploration, collecting, and social simulations were ranked above other game genres. These genres should be explored in regards to how they promote curiosity and whether they remain effective when introduced into games that emphasise other, lower ranked game genres.

The results of our study suggest that what makes players curious in a game does not necessarily correspond to their scores on the Five-Dimensional Curiosity Scale (5DC), although we caution that this finding is not conclusive. The closest connection was found in social curiosity, which corresponded to higher ranking of social simulation games. We also note that role-playing games were found to correlate with several dimensions of curiosity, suggesting that this game genre is able to invoke curiosity in different ways.

Through this study we have gathered valuable data for further research into how digital games invoke curiosity in players. We will expand on this work through an analysis of individual games to identify design patterns and related choices that are conductive to eliciting curiosity. This investigation should aid in the formulation of guidelines to design for curiosity. Ultimately, by fostering the development of games that intentionally invoke curiosity, we may be able to increase an individual's disposition to become curious. Such games would also provide interactive environments through which curiosity and related behaviour can be studied in the laboratory. Whether this potential can be fulfilled remains to be seen, but we hope that our work inspires continued exploration of this topic. 


\section{Acknowledgements}

We would like to thank Max van Duijn and Stefano Gualeni for their insight and support in carrying out this research. We further want to thank all of our participants for their time and effort.

\section{References}

1. Berlyne, D.E.: A theory of human curiosity. British Journal of Psychology 45(3), 180-191 (1954)

2. Berlyne, D.E.: Conflict, Arousal, and Curiosity. McGraw-Hill Book Company (1960)

3. Clarke, R.I., Lee, J.H., Clark, N.: Why video game genres fail: A classificatory analysis. Games and Culture 12(5), 445-465 (2017)

4. Costikyan, G.: Uncertainty in games. Mit Press (2013)

5. Day, H.I., et al.: Intrinsic motivation: A new direction in education. (1971)

6. Dewey, J.: How We Think. Heath \& Co. (1910)

7. Grossnickle, E.M.: Disentangling curiosity: Dimensionality, definitions, and distinctions from interest in educational contexts. Educational Psychology Review 28(1), 23-60 (2016)

8. Herbrich, R., Minka, T., Graepel, T.: Trueskill(tm): A bayesian skill rating system. pp. 569-576. MIT Press (January 2007), https://www.microsoft.com/enus/research/publication/trueskilltm-a-bayesian-skill-rating-system/

9. Inan, I.: The philosophy of curiosity. Routledge (2013)

10. Jirout, J., Klahr, D.: Childrens scientific curiosity: In search of an operational definition of an elusive concept. Developmental Review 32(2), 125-160 (2012)

11. Kang, M.J., Hsu, M., Krajbich, I.M., Loewenstein, G., McClure, S.M., Wang, J.T.y., Camerer, C.F.: The wick in the candle of learning: Epistemic curiosity activates reward circuitry and enhances memory. Psychological Science 20(8), 963-973 (2009)

12. Kashdan, T.B., Roberts, J.E.: Trait and state curiosity in the genesis of intimacy: Differentiation from related constructs. Journal of Social and Clinical Psychology 23(6), 792 (2004)

13. Kashdan, T.B., Stiksma, M.C., Disabato, D.D., McKnight, P.E., Bekier, J., Kaji, J., Lazarus, R.: The five-dimensional curiosity scale: Capturing the bandwidth of curiosity and identifying four unique subgroups of curious people. Journal of Research in Personality 73, 130-149 (2018)

14. Klimmt, C.: Dimensions and determinants of the enjoyment of playing digital games: A three-level model. In: Level up: Digital games research conference. pp. 246-257 (2003)

15. Kreitler, S., Zigler, E., Kreitler, H.: The nature of curiosity in children. Journal of school psychology 13(3), 185-200 (1975)

16. Lee, H.: Trueskill 0.4.4 python package. http://trueskill.org/ (2015)

17. Litman, J.A.: Interest and deprivation factors of epistemic curiosity. Personality and individual differences 44(7), 1585-1595 (2008)

18. Litman, J.A., Collins, R.P., Spielberger, C.D.: The nature and measurement of sensory curiosity. Personality and Individual Differences 39(6), 1123-1133 (2005)

19. Litman, J.A., Jimerson, T.L.: The measurement of curiosity as a feeling of deprivation. Journal of Personality Assessment 82(2), 147-157 (2004)

20. Litman, J.A., Silvia, P.J.: The latent structure of trait curiosity: Evidence for interest and deprivation curiosity dimensions. Journal of Personality Assessment 86(3), 318-328 (2006) 
21. Loewenstein, G.: The psychology of curiosity: A review and reinterpretation. Psychological Bulletin 116(1), 75 (1994)

22. McAuley, E., Duncan, T., Tammen, V.V.: Psychometric properties of the intrinsic motivation inventory in a competitive sport setting: A confirmatory factor analysis. Research quarterly for exercise and sport 60(1), 48-58 (1989)

23. Metacritic.com: Page: Best video games of all time. http://www.metacritic.com/browse/games/score/metascore/all/all/filtered (2018), accessed: 2018-01-31

24. Pluck, G., Johnson, H.: Stimulating curiosity to enhance learning. GESJ: Education Sciences and Psychology 2 (2011)

25. Reio, T.G., Callahan, J.L.: Affect, curiosity, and socialization-related learning: A path analysis of antecedents to job performance. Journal of Business and Psychology 19(1), 3-22 (2004)

26. Schaekermann, M., Ribeiro, G., Wallner, G., Kriglstein, S., Johnson, D., Drachen, A., Sifa, R., Nacke, L.E.: Curiously motivated: Profiling curiosity with self-reports and behaviour metrics in the game destiny. In: Proceedings of the Annual Symposium on Computer-Human Interaction in Play. pp. 143-156. ACM (2017)

27. Schmitt, F.F., Lahroodi, R.: The epistemic value of curiosity. Educational Theory $\mathbf{5 8}(2), 125-148$ (2008)

28. SeatGeek: Fuzzywuzzy. https://github.com/seatgeek/fuzzywuzzy (2017)

29. Sellke, T., Bayarri, M., Berger, J.O.: Calibration of $\rho$ values for testing precise null hypotheses. The American Statistician 55(1), 62-71 (2001)

30. Spielberger, C.D., Starr, L.M.: Curiosity and exploratory behavior. Motivation: Theory and research pp. 221-243 (1994)

31. To, A., Safinah, A., Kaufman, G.F., Hammer, J.: Integrating curiosity and uncertainty in game design. In: Proceedings of 1st International Joint Conference of DiGRA and FDG. pp. 1-16 (2016)

32. Yannakakis, G.N., Martínez, H.P.: Ratings are overrated! Frontiers in ICT 2, 13 (2015)

33. Zuckerman, M.: The sensation seeking scale v (sss-v): Still reliable and valid. Personality and Individual Differences 43(5), 1303-1305 (2007) 